\title{
Automatic Detection of Calcified Coronary Plaques in Computed Tomography Data Sets
}

\author{
Stefan C. Saur ${ }^{1}$, Hatem Alkadhi ${ }^{2}$, Lotus Desbiolles ${ }^{2}$, Gábor Székely${ }^{1}$, \\ and Philippe C. Cattin ${ }^{1,3}$ \\ ${ }^{1}$ Computer Vision Laboratory, ETH Zurich, Switzerland \\ saur@vision.ee.ethz.ch \\ ${ }^{2}$ Institute of Diagnostic Radiology, University Hospital Zurich, Switzerland \\ ${ }^{3}$ CMBE, University of Basel, Switzerland ${ }^{\star}$
}

\begin{abstract}
The detection of calcified plaques is an essential step in the assessment of coronary heart diseases. However, manual plaque segmentation is subjected to intra- and inter-observer variability. We present a novel framework for the automatic detection of calcified coronary plaques in Computed Tomography images. In contrast to the state-of-the-art, both the native and the angio data sets are included to gain additional information about each plaque for its detection and subsequent assessment. The framework was successfully tested on 127 patients where $85.5 \%$ of the calcified and $96 \%$ of the obstructive plaques have been detected.
\end{abstract}

\section{Introduction}

Coronary heart diseases (CHD) are a major cause of death within the United States 1 and other western countries. Early detection and quantification of coronary plaques is therefore of high interest. Depending on the degree of stenosis and the patient's health state, several treatment options exist: a coronary artery bypass graft (CABG), a drug eluting stent, or balloon angioplasty. Computed Tomography (CT) is a frequently used modality for the detection of plaques as it shows a good correlation with the gold standard Intravascular Ultrasound [2] and is, apart from the X-ray dose, not invasive. The standard scanning protocol for plaque assessment consists of two scans: a native, low resolution scan and a higher resolution angio scan with contrast agent. The first one is mainly used for Calcium Scoring as calcified plaques are clearly visible. The latter nicely visualizes the vessel lumen and as such allows to assess the degree of a stenosis. It furthermore allows to localize and characterize soft plaques.

For the automatic detection of plaques, it is helpful to delineate the coronary arteries, i.e. determining the centerline or a full segmentation of the vessel lumen. A comprehensive overview of existing segmentation algorithms for tubular structures can be found in [3]. With the various branches of the coronary

\footnotetext{
* This work has been supported by the CO-ME/NCCR research network of the Swiss National Science Foundation (http://co-me.ch). The authors thank MeVis Research (Bremen, Germany) for their support.
}

D. Metaxas et al. (Eds.): MICCAI 2008, Part I, LNCS 5241, pp. 170-177, 2008.

(C) Springer-Verlag Berlin Heidelberg 2008 
artery tree segmented, it is possible to analyze each branch for the presence of plaques. Toumoulin et al. 4] used a level set approach to detect both the inner and outer wall of a vessel in CT data sets and were therefore able to identify calcified plaques. Rinck et al. 5] also computed the inner and outer vessel wall to assess atherosclerotic plaques in coronary arteries using shape-based segmentation. Their algorithm, however, had to be manually initialized with a seed point before and after each plaque. Išgum et al. [6] applied a heart and aorta segmentation to native data sets and used specific features to automatically detect coronary calcifications using a two-stage classification system with a k-NN classifier and a feature selection scheme. They could detect $73.8 \%$ of the calcified plaques at the expense of an average of 0.1 false positives per scan. A 'Plaque Map' for CT images that uses color-based isometric lines and a bird's eye view to support the plaque detection was introduced in [7. However, this method is only a visual aid for the manual detection of plaques. A model-based approach to semi-automatically measure the degree of stenosis in carotid arteries was developed by Frangi et al. 8, for MR images. Adame et al. 9] combined model-based segmentation and fuzzy clustering to detect the vessel wall, lumen and lipid core boundaries in MR images. Also for MR images, Sun et al. [10] used a Fuzzy C-Means based clustering algorithm to characterize plaque constituents.

With one exception, all aforementioned methods require substantial user intervention. The approach proposed in 6] is fully automatic but only detects calcified plaques in native data sets for Calcium Scoring. In this paper, we propose a framework for the automatic detection of calcified coronary plaques. In contrast to the previous techniques, we incorporate both the angio and native data set. Using both data sets has the advantage that more information for each plaque is available, both for its detection and subsequent assessment.

\section{Method}

This section presents a framework for the automatic detection of coronary calcified plaques. As in general both the angio and native CT scans are acquired for the assessment of plaques, no additional scan is required for the application of the proposed method. The basic concept can be summarized as follows. In the pre-processing steps $(\mathrm{A})+(\mathrm{B})$ the aorta is detected and the coronary tree is segmented. The third step (C) extracts putative plaque candidates using an intensity-based threshold. This results in many false positives (FP) due to noise, inhomogeneous contrast agent distribution and other CT artifacts. In step (D) the plaques in the angio data set with the highest calcification are rigidly registered to the native plaques. The last two steps $(\mathrm{E})+(\mathrm{F})$ use a rule based approach to maximize the number of detected plaques while minimizing FP. Throughout the processing pipeline, four sets are used to represent the actual state of the detection process: (1) $\mathcal{A}$ contains the unverified angio plaque candidates, (2) $\mathcal{N}_{130}$ the unverified native plaque candidates, (3) $\mathcal{N}_{200} \subset \mathcal{N}_{130}$ is a subset of $\mathcal{N}_{130}$ and contains the highly calcified but still unverified plaques only and (4) the initially empty set $\mathcal{V}$ contains the verified plaques. 
(A) Aorta Detection. A point $\boldsymbol{p}_{\text {aorta }}$ in the ascending aorta is automatically detected in the angio data set [1] and the voxels in a $7 \times 7 \times 7$ neighborhood around $\boldsymbol{p}_{\text {aorta }}$ are used to compute the mean Hounsfield (HU) intensity $\mu$ and standard deviation $\sigma$ of the contrast agent. These parameters are then later used to automatically select the threshold for the plaque candidate extraction.

(B) Coronary Artery Tree. The rough estimate of the vessel's centerline is obtained with a livewire algorithm [12, using only the intensity and gradient features as costs. Seed points need to be manually placed at the orifices of the coronary arteries from the aorta and in the distal ends of the various branches. The livewire algorithm was further modified to automatically detect bifurcations to extract the branching information of the coronary artery tree. For this, the centerline starting at the currently processed distal seed point may terminate either in one of the seed points at the orifices or in an existing centerline generated by previously processed seed points. The starting and terminal points of each centerline are kept for the subsequent lumen segmentation. The vessel lumen is segmented using graphcut (GC) 13. For this, each detected branch is reformatted along its centerline to get a stack of cross-sectional images. GC is then applied to each cross-sectional slice using a circular shape prior [14. The resulting segmentation mask is then transformed back into the original angio data set.

(C) Candidate extraction. Plaque candidates are extracted using an intensity threshold from both the angio and the native data set.

Angio data set. The coronary artery segmentation of the previous step is used to restrict the plaque candidates to only those within the coronary arteries. The subsequent marching cube algorithm then generates meshes of all putative plaques. The iso-surface value $(\mu+5 \sigma)$ is adaptively chosen according to the contrast agent density observed in the aorta. Each connected mesh is regarded as an angio plaque candidate $a_{i}$ which is stored in the list $\mathcal{A}$. Each candidate $a_{i}$ is additionally assigned an intensity score $s$ depending on its $90 \%$-quantile intensity $I$ of its voxels and defined by

$$
s=\left\{\begin{aligned}
0 & : \quad I<\mu+2 \sigma \\
\log _{10}\left(9 \frac{I-(\mu+2 \sigma)}{500 \mathrm{HU}-(\mu+2 \sigma)}+1\right) & : \quad \mu+2 \sigma \leq I<500 \mathrm{HU} \\
1 & : I>500 \mathrm{HU}
\end{aligned}\right.
$$

The 90\%-quantile for the intensity $I$ was chosen to avoid outliers stemming from CT artifacts. The intensity score is defined for values $I \geq \mu+2 \sigma$ to potentially include extremely weak calcified plaques. However, those are currently not extracted and processed in the proposed framework.

Native Data Set. A radiation absorption intensity threshold of $t h_{i}=130 \mathrm{HU}$ is applied to the native data set. To ignore most of the bones, connected components larger than $5000 \mathrm{~mm}^{3}$ are discarded. The resulting binary image is used as a mask for the native data set and the meshes of all plaques with an iso-surface value of $130 \mathrm{HU}$ are then extracted using again the marching cube algorithm. 
Each connected mesh is regarded as a native plaque candidate $n_{i}$ and is stored in the list $\mathcal{N}_{130}$. The same procedure is repeated but with $t h_{i}=200 \mathrm{HU}$, to generate a set of higher calcified native candidates called $\mathcal{N}_{200}$.

(D) Plaque registration. Although the native and angio data sets are acquired consecutively, they are generally not well registered due to the beating heart, breathing and minor patient movement. It is therefore the aim of the rigid plaque registration to determine the rotation $\boldsymbol{R}$ and translation $\boldsymbol{T}$ between both data sets. This registration allows to take over the coronary segmentation results from the angio to the native data set such that the native candidates can also be limited to the segmented vessel regions. Furthermore, it enables to compare plaque features from both data sets with each other. To avoid misregistrations, only the very high calcified native plaques of $\mathcal{N}_{200}$ as well as the angio plaques with a score $s>0.95$ and a volume $v>5$ voxels are considered for registration. As due to the different scan resolutions the shape between corresponding angio and native candidates may differ, only the barycenters of their meshes are used during the two stage registration process. These barycenters are stored in the list $\mathcal{R}_{\mathrm{N}}$ for the native and $\mathcal{R}_{\mathrm{A}}$ for the angio candidates, respectively. Misregistrations might occur if a large plaque in the low resolution native data set in fact consists of multiple smaller plaques in the angio data set. To resolve this ambiguity, additional points are added to $\mathcal{R}_{\mathrm{N}}$ by sampling all native plaques with an extension greater than $4 \mathrm{~mm}$. Starting from the barycenter, additional points are placed with a step width of $\Delta=2 \mathrm{~mm}$ in both directions along the plaque's main axis as long as they are situated within the plaque (Fig. 17a).

In the first stage of the rigid registration, an optimal translation $\boldsymbol{t}^{*}$ is searched. The rotation is disregarded in this phase as according to our experience it has only a minor impact on the final registration. The optimal translation $\boldsymbol{t}^{*}$ is obtained by first choosing an arbitrary point from $\mathcal{R}_{\mathrm{A}}$ as a reference $a_{\text {ref }}$. Then, for each point $p_{n} \in \mathcal{R}_{\mathrm{N}}$, the translation $\boldsymbol{t}=\boldsymbol{p}_{\boldsymbol{n}}-\boldsymbol{a}_{\text {ref }}$ is computed (Fig. 1 $\mathrm{b}$ ) and applied to $\mathcal{R}_{\mathrm{A}}$ resulting in $\mathcal{R}_{\mathrm{A}^{\prime}}$ (Fig. 1 1 ). Correspondences between angio and native candidates are established by mapping each transformed $p_{a_{i}^{\prime}} \in \mathcal{R}_{\mathrm{A}^{\prime}}$ to the point $p_{n_{i}} \in \mathcal{R}_{\mathrm{N}}$ with the smallest euclidean distance (Fig. 1F). Afterwards, the energy $E_{p_{n}}=E_{1}+E_{2}+E_{3}$ is computed. The first energy term $E_{1}=\|\boldsymbol{t}\|$ accounts for the length of $\boldsymbol{t}$ which should be minimal as only a small heart motion between the angio and native data set is expected. The second term $E_{2}=\sum_{i}\left\|\boldsymbol{p}_{\boldsymbol{a}_{\boldsymbol{i}}^{\prime}}-\boldsymbol{p}_{\boldsymbol{n}_{\boldsymbol{i}}}\right\|$ considers the remaining distance between the angio and native barycenters of corresponding candidate pairs. The third energy term $E_{3}$ compares how similar the spatial distribution of the angio candidates is with the spatial distribution of the mapped native candidates. For this, the distance $e_{a_{i j}}=\left\|\boldsymbol{p}_{\boldsymbol{a}_{\boldsymbol{i}}}-\boldsymbol{p}_{\boldsymbol{a}_{j}}\right\|$ of any possible combination of two different points $p_{a_{i}}, p_{a_{j}} \in \mathcal{R}_{\mathrm{A}}, i \neq j$ is compared with the distance $e_{n_{i j}}=\left\|\boldsymbol{p}_{\boldsymbol{n}_{\boldsymbol{i}}}-\boldsymbol{p}_{\boldsymbol{n}_{\boldsymbol{j}}}\right\|$ of the corresponding mapped native candidates. The energy $E_{3}$ is then defined as $E_{3}=\frac{1}{n} \sum\left|e_{a_{i j}}-e_{n_{i j}}\right|$ where $n$ is the total number of possible combinations.

In the second stage, the known mapping of angio-native pairs with the minimum energy $E_{p_{n}}$ is taken to compute the final registration parameters $\boldsymbol{R}$ and $\boldsymbol{T}$, using the algorithm from Horn [15] which requires at least three point pairs. For fewer 
pairs, $\boldsymbol{R}$ is assumed to be the unity matrix. $\boldsymbol{T}$ is set to $\frac{1}{2}\left(\left(\boldsymbol{p}_{\boldsymbol{a}_{1}}-\boldsymbol{p}_{\boldsymbol{n}_{\mathbf{1}}}\right)+\left(\boldsymbol{p}_{\boldsymbol{a}_{\mathbf{2}}}-\boldsymbol{p}_{\boldsymbol{n}_{\mathbf{2}}}\right)\right)$ for two, to $\boldsymbol{p}_{\boldsymbol{a}_{1}}-\boldsymbol{p}_{\boldsymbol{n}_{1}}$ for one, and to the null vector if no plaque pair is present. The rotation $\boldsymbol{R}$ and translation $\boldsymbol{T}$ are then applied to all native candidates from $\mathcal{N}_{130}$. Those falling outside of the vessel boundaries after the registration are discarded. All matched angio-native candidate pairs are added to $\mathcal{V}$ and removed from $\mathcal{A}$ and $\mathcal{N}_{130}$, respectively.

(E) Distance rule. Several distance checks are applied to confirm the verified candidates resulting from the registration process and to search for additional pairs that were not considered by the registration process due to their low intensity. Outliers in the registration can occur if a plaque is visible in the angio data set but not in the native acquisition. Then, the corresponding plaque is mapped to a wrong - in general more distant - candidate. Therefore, all previously verified angio-native candidate pairs in $\mathcal{V}$ are re-evaluated. If the distance between the angio and native plaque is larger than $0.5 \mathrm{~mm}$, the candidate pair is removed from $\mathcal{V}$ and put back to $\mathcal{A}$ or $\mathcal{N}_{130}$, respectively. Additional angionative candidate pairs are detected by iterating through $\mathcal{A}$ and checking for each angio candidate $a_{i}$ if there is a native plaque $n_{i}$ within a distance of $0.5 \mathrm{~mm}$. If yes, this pair is added to $\mathcal{V}$ and removed from $\mathcal{A}$ and $\mathcal{N}_{130}$.

(F) Intensity score rule. This rule considers the fact that large angio plaque candidates with high intensity values are very likely to be plaques even though a corresponding native candidate is missing. An angio candidate is added to $\mathcal{V}$ and removed from $\mathcal{A}$ if its volume is larger than $1 \mathrm{~mm}^{3}$ and $s \geq 0.75$.

\section{Results}

The proposed framework was developed in $\mathrm{C}++$ based on the platform MeVisLab (MeVis Bremen, Germany) and evaluated on 127 patients (81 males, 46 females, mean age $63.8 \pm 12.0$, range $35-88$ ). To guarantee robustness, only the first 20 patients were used for development and parameter selection. Both angio and native scans were acquired on a Dual-Source CT scanner (Somatom Definition, Siemens Medical Solutions, Forchheim, Germany) with standard protocols. The in-plane resolution ranged from $0.25-0.73 \mathrm{~mm}(512 \times 512$ voxels $)$. The slice thickness/slice spacing was $0.75-1 \mathrm{~mm} / 0.4-1 \mathrm{~mm}$ for the angio and $3 \mathrm{~mm} / 1.5-3 \mathrm{~mm}$ for the native data sets. The number of slices varied between 115 and 392 for the angio and between 26 and 101 for the native acquisitions, respectively.

The ground truth was obtained by manual plaque segmentation by a radiologist. The type of plaque (calcified or mixed), the degree of stenosis and the proximal and distal end position of each plaque were determined. A plaque with a stenosis greater than $50 \%$ was regarded as obstructive which was observed for 54 plaques (41 calcified, 13 mixed). In total 649 calcified and 102 mixed plaques were labeled. Figure 2 shows the allocation of these plaques over the patients. Besides the 33 cases with no plaques, most patients had between 1-8 plaques. 


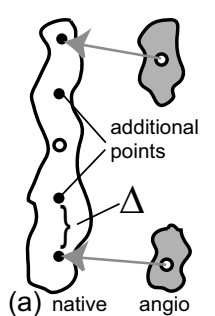

(a) native angio

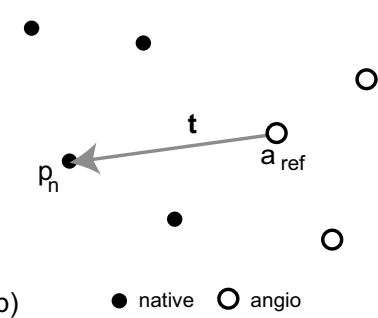

(b)

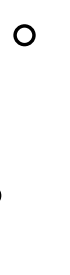

(c)

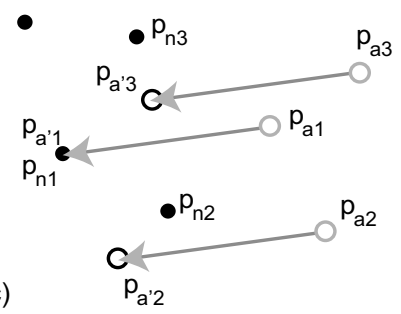

Fig. 1. Plaque registration. (a) Additional points are added for large native plaque candidates. (b) Reference point $a_{\text {ref }}$ and translation vector $\boldsymbol{t}$ (c) Translation and mapping of angio candidates $p_{a_{i}^{\prime}}$ to closest native candidates $p_{n_{i}}$.

Table 1. Number (percentage) of detected calcified and mixed plaques as well as the number of true (TP) and false (FP) positives, and the positive predictive value (PPV) for the candidates of the detection framework after each processing step. Some larger plaques are represented by multiple smaller candidates such that the number of TP and detected plaques is not identical.

\begin{tabular}{|r|r|r|r|r|r|}
\hline & expert & $\begin{array}{r}\text { candidate } \\
\text { extraction }\end{array}$ & $\begin{array}{r}\text { plaque } \\
\text { registration }\end{array}$ & $\begin{array}{r}\text { distance } \\
\text { rule }\end{array}$ & $\begin{array}{r}\text { intensity } \\
\text { score rule }\end{array}$ \\
\hline plaques calcified & 649 & $594(91.5 \%)$ & $436(67.2 \%)$ & $497(76.6 \%)$ & $560(86.3 \%)$ \\
mixed & 102 & $85(83.3 \%)$ & $66(64.7 \%)$ & $75(73.5 \%)$ & $82(80.4 \%)$ \\
\hline candidates TP & 751 & 760 & 544 & 644 & 723 \\
FP & 0 & 699 & 50 & 59 & 100 \\
PPV & $100.0 \%$ & $52.1 \%$ & $91.6 \%$ & $91.6 \%$ & $87.8 \%$ \\
\hline
\end{tabular}

The verified plaque candidates from $\mathcal{V}$ were compared with the ground truth data to determine the number of true (TP) and false positives (FP). The number of detected labeled plaques as well as the positive predictive value $P P V=$ $T P /(F P+T P)$ after each processing step are summarized in Table1 The framework detected $86.3 \%$ of the calcified and $80.4 \%$ of the mixed plaques which leads to a total detection rate of $85.5 \%$ with a PPV of $87.8 \%$. In total 109 (89 calcified, 20 mixed) plaques were missed. The mean extension $\bar{d}_{e}$ of the missed calcified and mixed plaques was $1.3 \mathrm{~mm}$ and $4.9 \mathrm{~mm}$, respectively. One small obstructive calcified $\left(\mathrm{d}_{\mathrm{e}}=1.2 \mathrm{~mm}\right)$ and one obstructive mixed plaque $\left(\mathrm{d}_{\mathrm{e}}=3.4 \mathrm{~mm}\right)$ were not detected, resulting in a detection rate of $96 \%$ for the hazardous obstructive plaques. The extent of mixed plaques includes both the lipid and calcified part. Therefore, those missed plaques appear larger although only the small calcified part could not be detected. Figure $2 \mathrm{~b}$ shows an example of detected angio-native plaque candidate pairs before the registration is applied to the native candidates.

\section{Discussion and Conclusion}

We presented a framework for the automatic detection of calcified coronary plaques. Through the proposed novel integration of both the native and the 
angio data sets into the detection process, we were able to achieve a good detection rate of $85.5 \%$. However, a direct comparison to the method proposed by Išgum et al. [6] would not be feasible for several reasons: First of all, we encountered with 0.79 a higher average number of FP per patient. We assume that the detection rate from Išgum et al. 6] would improve if a comparable number of FP would be allowed. Further, the study population of Išgum et al. [6] consisted of women in their menopause whereas our population was a subset of a typical population scheduled for CT Coronary Angiography. Išgum et al. 6] only detected calcified coronary plaques in the native data set for Calcium Scoring, whereas our presented framework identifies the plaques both in the native and angio data set and establishes correspondences among them. Through this it will become possible to provide detailed information, i.e. both the Calcium Score and the degree of stenosis for each plaque individually.

An analysis of the FP has shown that they accumulate only in a small number of 37 patients (Fig 2 ) where they mostly arose due to an inhomogeneous contrast agent distribution within the distal part of the right coronary artery. The not detected plaques were generally small and with two exceptions non-obstructive. In general, those small plaques did not have a major impact on the diagnoses as a physician mainly looks for stenoses or large plaques.

Although, many plaques could be detected by applying an intensity-based threshold, this would result in a high number of FP. Therefore, we added additional steps to verify the extracted plaque candidates. The registration step alone significantly reduced the number of FP. This is obvious as only highly calcified plaques were included. It also explains the decreased detection rate compared to the previous step. Additional criterions for the evaluation of the remaining weaker calcified plaque candidates could considerably improve the detection rate, nevertheless eventually at the expense of increased FP rate.

The good performance of the proposed approach can be mainly attributed to the fusion of the native and angio data set. This allowed to carry over the segmented vessels into the native data set and thereby reducing the FP rate.

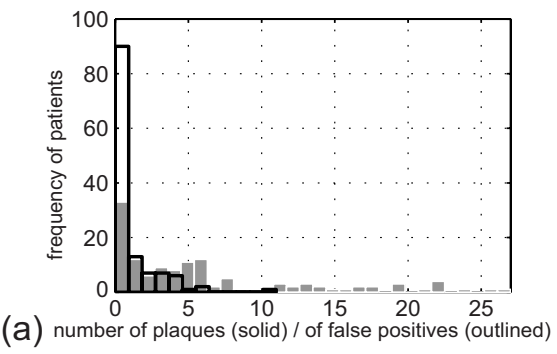

(b)

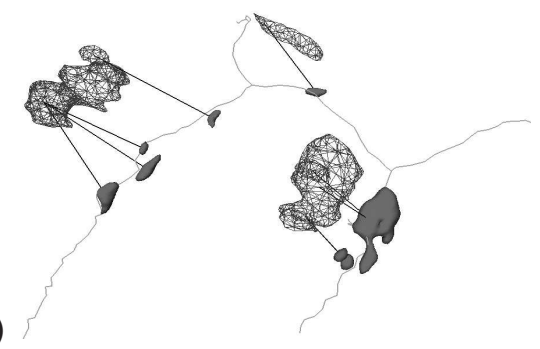

Fig. 2. (a) Histogram showing the distribution of the number of plaques per patient (solid bars) and the distribution of false positives (FP) accounted per patient (outlined bars). (b) Example of established correspondences between angio $a_{i}$ (solid) and native candidates $n_{i}$ (mesh) before the registration is applied to the native candidates. 
Moreover, weakly calcified plaques, hardly discriminable from the contrast agent in the angio data set, could be detected. The approach still offers a wide range of possible improvements that can be easily integrated due to its modular design. In particular we are working on methods to automatically classify the detected plaques and to estimate the degree of stenosis. Furthermore, we are investigating methods to automatically label the segments of the coronary artery tree. This would allow for the fully automatic generation of a detailed medical report.

\section{References}

1. Rosamond, W., et al.: Heart Disease and Stroke Statistics-2008 Update. Circulation 117(4), 25-146 (2008)

2. Achenbach, S., et al.: Detection of Calcified and Noncalcified Coronary Atherosclerotic Plaque by Contrast-Enhanced, Submillimeter Multidetector Spiral Computed Tomography. Circulation 109(1), 14-17 (2004)

3. Kirbas, C., Quek, F.: A Review of Vessel Extraction Techniques and Algorithms. ACM Comput. Surv. 36(2), 81-121 (2004)

4. Toumoulin, C., et al.: Coronary Characterization in Multi-slice Computed Tomography. In: Comput. Cardiol., pp. 749-752 (2003)

5. Rinck, D., et al.: Shape-based Segmentation and Visualization Techniques for Evaluation of Atherosclerotic Plaques in Coronary Artery Disease. In: SPIE (2006)

6. Išgum, I., Rutten, A., Prokop, M., van Ginneken, B.: Detection of coronary calcifications from computed tomography scans for automated risk assessment of coronary artery disease. Med. Phys. 34(4), 1450-1461 (2007)

7. Komatsu, S., et al.: Detection of Coronary Plaque by Computed Tomography With a Novel Plaque Analysis System, 'Plaque Map', and Comparison With Intravascular Ultrasound and Angioscopy. Circulation 69(1), 72-77 (2005)

8. Frangi, A., Niessen, W., Nederkoorn, P., et al.: Three-Dimensional Model-Based Stenosis Quantification of the Carotid Arteries from Contrast-Enhanced MR Angiography. In: MMBIA, pp. 110-118 (2000)

9. Adame, I.M., et al.: Automatic segmentation and plaque characterization in atherosclerotic carotid artery MR images. MAGMA 16(5), 227-234 (2004)

10. Sun, B., et al.: Automatic Plaque Characterization Employing Quantitative and Multicontrast MRI. Magnetic Resonance in Medicine 59(1), 174-180 (2008)

11. Saur, S., et al.: Automatic Ascending Aorta Detection in CTA Datasets. In: Bildverarbeitung für die Medizin, pp. 323-327 (2008)

12. Barrett, W.A., Mortensen, E.N.: Interactive live-wire boundary extraction. Medical Image Analysis 1(4), 331-341 (1997)

13. Boykov, Y., Jolly, M.P.: Interactive Graph Cuts for Optimal Boundary \& Region Segmentation of Objects in N-D Images. In: ICCV, vol. 1, pp. 105-112 (2001)

14. Slabaugh, G., Unal, G.: Graph cuts segmentation using an elliptical shape prior. In: ICIP, vol. 2, pp. 1222-1225 (2005)

15. Horn, B.K.P.: Closed-form solution of absolute orientation using unit quaternions. Journal of the Optical Society of America 4, 629-642 (1987) 